\title{
Internal labelling operators and contractions of Lie algebras
}

\author{
R. Campoamor-Stursberg $\dagger$ \\ † Dpto. Geometría y Topología \\ Fac. CC. Matemáticas \\ Universidad Complutense de Madrid \\ Plaza de Ciencias, 3 \\ E-28040 Madrid, Spain \\ E-mail: rutwig@mat.ucm.es
}

\begin{abstract}
We analyze under which conditions the missing label problem associated to a reduction chain $\mathfrak{s}^{\prime} \subset \mathfrak{s}$ of (simple) Lie algebras can be completely solved by means of an Inönü-Wigner contraction $\mathfrak{g}$ naturally related to the embedding. This provides a new interpretation of the missing label operators in terms of the Casimir operators of the contracted algebra, and shows that the available labeling operators are not completely equivalent. Further, the procedure is used to obtain upper bounds for the number of invariants of affine Lie algebras arising as contractions of semisimple algebras.
\end{abstract}

PACS numbers: $02.20 \mathrm{~Sv}, 02.20 \mathrm{Qs}$ 


\section{Introduction}

A recurring problem in group theoretical applications to physical problems is the reduction of irreducible representations of a Lie group into multiplets of some subgroup of internal symmetry. Sometimes, and depending on the nature of the embedding, the subgroup does not provide enough labels to distinguish the basis states without ambiguity. We are therefore led to find additional operators to separate those states not properly described by the subgroup labels. Various techniques have been developed to surmount this difficulty, such as the projection technique of Elliott for the reduction chain $\mathfrak{s u}(3) \supset \mathfrak{s o}(3)$ used in atomic physics, the method of elementary multiplets in the spectroscopic chain $\mathfrak{s o}(7) \supset G_{2} \supset \mathfrak{s o}(3)$ to describe $f$ electron configurations of rare earths, or the construction of integrity bases in the enveloping algebras for the Wigner supermultiplet model $\mathfrak{s u}(4) \supset \mathfrak{s u}(2) \times \mathfrak{s u}(2)$, among others [1]. More recently, K-matrix theory and the rotor expansion method have been shown to be powerful techniques to solve the missing label problem in many important problems, like the nuclear $\mathfrak{s p}(3)$ model [2, 3].

A complementary analytical approach to the so-called missing label problem (MLP) was developed in [4, 5], by means of basis functions that are common eigenstates of commuting operators. This point of view also allows to recover the missing operators as subgroup scalars in the enveloping algebra of $\mathfrak{s}$, as well as to compute them as solutions of a system of partial differential equations. Although this approach has been the less used for solving the MLP, it presents some interesting features over the pure algebraic method of enveloping algebras. It has been observed in the literature that symmetry breaking is, to some extent, equivalent to consider contractions of Lie algebras [6]. In this sense, the symmetry preserved corresponds to some subalgebra which remains unchanged by the contraction. At least for the $\mathfrak{s u}(3)$ model, this idea has been developed by means of the rotor expansion [3].

This is the point of view we adopt in this work. More specifically, we combine the analytical method of [5] for solving the MLP with contractions of Lie algebras. We prove that for any embedding $\mathfrak{s} \supset \mathfrak{s}^{\prime}$ of (semisimple) Lie algebras, there is an associated simple Inönü-Wigner contraction of $\mathfrak{s}$ onto an affine Lie algebra $\mathfrak{g}=\mathfrak{s}^{\prime} \vec{\oplus}_{R} n L_{1}$, where $n L_{1}$ denotes an $n$-dimensional Abelian algebra and $R$ is a representation of the subalgebra $\mathfrak{s}^{\prime}$ such that the adjoint representation $\operatorname{ad}$ of $\mathfrak{s}$ satisfies the condition $\operatorname{ad}(\mathfrak{s})=\operatorname{ad}\left(\mathfrak{s}^{\prime}\right) \oplus R$. It is further proven that any invariant of the contraction $\mathfrak{g}$ can be formally taken as missing label operator. It is therefore reasonable to study whether the invariants of the contraction $\mathfrak{g}$ are sufficient in number to provide a set of missing label operators, and therefore, to completely solve the missing label problem. We characterize when it is possible to solve the MLP by means of this associated contraction, and derive some useful consequences for the number of invariants of inhomogeneous Lie algebras. One important fact arises from this method, namely, that the missing label operators obtained inherit an intrinsic meaning as terms of invariants that disappear during contraction, and should correspond to the natural choice of operators, since they are 
internally determined by the group-subgroup chain. For the case of no missing labels, we extract an interesting consequence, namely, that the invariants of the contraction arise as polynomial functions of the Casimir operators of the contracted Lie algebra $\mathfrak{s}$ and the subalgebra $\mathfrak{s}^{\prime}$. This enables us to determine upper bounds for the number of inhomogeneous Lie algebras that appear as contractions of semisimple Lie algebras.

It is known from the classical theory that irreducible representations of semisimple Lie algebras are labelled unambigously by the eigenvalues of Casimir operators. More generally, it can be established that irreducible representations of a Lie algebra $\mathfrak{g}$ are labelled using the eigenvalues of its generalized Casimir invariants [5]. The number of internal labels needed equals

$$
i=\frac{1}{2}(\operatorname{dim} \mathfrak{g}-\mathcal{N}(\mathfrak{g}))
$$

as first observed by Racah [7]. If we use some subalgebra $\mathfrak{h}$ to label the basis states of $\mathfrak{g}$, we obtain $\frac{1}{2}\left(\operatorname{dim} \mathfrak{h}+\mathcal{N}(\mathfrak{h})+l^{\prime}\right.$ labels, where $l^{\prime}$ is the number of invariants of $\mathfrak{g}$ that depend only on variables of the subalgebra $\mathfrak{h}[5]$. In order to separate irreducible representations of $\mathfrak{g}$ uniquely, it is necessary to find

$$
n=\frac{1}{2}(\operatorname{dim} \mathfrak{g}-\mathcal{N}(\mathfrak{g})-\operatorname{dim} \mathfrak{h}-\mathcal{N}(\mathfrak{h}))+l^{\prime}
$$

additional operators, which are usually called missing label operators. The total number of available operators of this kind is easily shown to be twice the number of needed labels, i.e., $m=2 n$. For $n>1$, it remains the problem of determining a set of $n$ mutually commuting operators. The analytical approach to the missing label problem has the advantage of pointing out its close relation to the problem of finding the invariants of the coadjoint representation of a Lie algebra. Although in general the missing label operators do not constitute invariants of the algebra or subalgebra, they can actually be determined with the same Ansatz [5, 8, 9]. Given the Lie algebra $\mathfrak{g}$ with structure tensor $\left\{C_{i j}^{k}\right\}$ over a basis $\left\{X_{1}, . ., X_{n}\right\}$, we realize the algebra in the space $C^{\infty}\left(\mathfrak{g}^{*}\right)$ by means of the differential operators defined by:

$$
\widehat{X}_{i}=C_{i j}^{k} x_{k} \frac{\partial}{\partial x_{j}},
$$

where $\left[X_{i}, X_{j}\right]=C_{i j}^{k} X_{k} \quad(1 \leq i<j \leq n)$ and $\left\{x_{1}, . ., x_{n}\right\}$ is a dual basis of $\left\{X_{1}, . ., X_{n}\right\}$. The invariants of $\mathfrak{g}$ (in particular, the Casimir operators) are solutions of the following system of partial differential equations:

$$
\widehat{X}_{i} F=0, \quad 1 \leq i \leq n .
$$

Whenever we have a polynomial solution of (4), the symmetrization map defined by

$$
\operatorname{Sym}\left(x_{i_{1}}^{a_{1}} \ldots x_{i_{p}}^{a_{p}}\right)=\frac{1}{p !} \sum_{\sigma \in S_{p}} x_{\sigma\left(i_{1}\right)}^{a_{1}} . . x_{\sigma\left(i_{p}\right)}^{a_{p}}
$$

allows to recover the Casimir operators in their usual form, i.e, as elements in the centre of the enveloping algebra of $\mathfrak{g}$. A maximal set of functionally independent invariants is usually called a fundamental basis. The number $\mathcal{N}(\mathfrak{g})$ of functionally independent 
solutions of (44) is obtained from the classical criteria for differential equations, and is given by:

$$
\mathcal{N}(\mathfrak{g}):=\operatorname{dim} \mathfrak{g}-\operatorname{rank}\left(C_{i j}^{k} x_{k}\right),
$$

where $A(\mathfrak{g}):=\left(C_{i j}^{k} x_{k}\right)$ is the matrix associated to the commutator table of $\mathfrak{g}$ over the given basis. If we now consider an algebra-subalgebra chain $\mathfrak{s} \supset \mathfrak{s}^{\prime}$ determined by the embedding $f$, in order to compute the missing label operators we have to consider the equations of (4) corresponding to the generators of the subalgebra $\mathfrak{s}^{\prime}$. This system, as proven in [5], has exactly $\mathcal{N}\left(f\left(\mathfrak{s}^{\prime}\right)\right)=\operatorname{dim} \mathfrak{s}-\operatorname{dim} \mathfrak{s}^{\prime}-l^{\prime}$ solutions. Using formula (2) it follows further that this scalar can be expressed in terms of the number of invariants of the algebra-subalgebra chain:

$$
\mathcal{N}\left(f\left(\mathfrak{s}^{\prime}\right)\right)=m+\mathcal{N}(\mathfrak{s})+\mathcal{N}\left(\mathfrak{s}^{\prime}\right)-l^{\prime} .
$$

This shows that the differential equations corresponding to the subalgebra generators have exactly $n$ more solutions as needed to solve the missing label problem. We remark that the scalar $m$ depends essentially on the embedding $f$.

Since we are interested in combining the invariants with contractions, we briefly recall the elementary notions that will be used in the following. Let $\mathfrak{g}$ be a Lie algebra and $\Phi_{t} \in \operatorname{End}(\mathfrak{g})$ a family of non-singular linear maps, where $t \in[1, \infty) \biguplus$ For any $X, Y \in \mathfrak{g}$ we define

$$
[X, Y]_{\Phi_{t}}:=\Phi_{t}^{-1}\left[\Phi_{t}(X), \Phi_{t}(Y)\right]
$$

which obviously represent the brackets of the Lie algebra over the transformed basis. Now suppose that the limit

$$
[X, Y]_{\infty}:=\lim _{t \rightarrow \infty} \Phi_{t}^{-1}\left[\Phi_{t}(X), \Phi_{t}(Y)\right]
$$

exists for any $X, Y \in \mathfrak{g}$. Then equation (9) defines a Lie algebra $\mathfrak{g}^{\prime}$ called the contraction of $\mathfrak{g}$ (by $\Phi_{t}$ ), non-trivial if $\mathfrak{g}$ and $\mathfrak{g}^{\prime}$ are non-isomorphic, and trivial otherwise [10, 11]. A contraction for which there exists some basis $\left\{X_{1}, . ., X_{n}\right\}$ such that the contraction matrix $A_{\Phi}$ is diagonal, that is, adopts the form

$$
\left(A_{\Phi}\right)_{i j}=\delta_{i j} t^{n_{j}}, \quad n_{j} \in \mathbb{Z}, t>0
$$

is called a generalized Inönü-Wigner contraction [11]. This is the only type of contractions that we will need in this work. It is known (see e.g. [12]) that for a contraction $\mathfrak{g} \rightsquigarrow \mathfrak{g}^{\prime}$ of Lie algebras, the following inequality must be satisfied

$$
\mathcal{N}(\mathfrak{g}) \leq \mathcal{N}\left(\mathfrak{g}^{\prime}\right)
$$

The notion of contraction can also be formulated for invariant functions [13]. The procedure is formally valid for polynomial and non-polynomial invariants, but in this work we will only consider Casimir operators. Suppose that the contraction is of the $\ddagger$ Other authors use the parameter range $(0,1]$, which is equivalent to this by simply changing the parameter to $t^{\prime}=1 / t$. 
type (10). If $F\left(X_{1}, \ldots, X_{n}\right)=\alpha^{i_{1} \ldots i_{p}} X_{i_{1}} \ldots X_{i_{p}}$ is a Casimir operator of degree $p$, then the transformed invariant takes the form

$$
F\left(\Phi_{t}\left(X_{1}\right), . ., \Phi_{t}\left(X_{n}\right)\right)=t^{n_{i_{1}}+\ldots+n_{i_{p}}} \alpha^{i_{1} \ldots i_{p}} X_{i_{1}} \ldots X_{i_{p}} .
$$

Now, defining

$$
M=\max \left\{n_{i_{1}}+\ldots+n_{i_{p}} \mid \alpha^{i_{1} . i_{p}} \neq 0\right\}
$$

the limit

$F^{\prime}\left(X_{1}, . ., X_{n}\right)=\lim _{t \rightarrow \infty} t^{-M} F\left(\Phi_{t}\left(X_{1}\right), \ldots, \Phi_{t}\left(X_{n}\right)\right)=\sum_{n_{i_{1}}+\ldots+n_{i_{p}}=M} \alpha^{i_{1} \ldots i_{p}} X_{i_{1}} \ldots X_{i_{p}}$

gives a Casimir operator of degree $p$ of the contraction $\mathfrak{g}^{\prime}$. It should be remarked that, starting from an adequate fundamental system of invariants $\left\{C_{1}, . ., C_{p}\right\}$ of $\mathfrak{g}$, it is always possible to obtain a set of $p$ independent invariants of the contraction. However, it is not ensured that these invariants are of minimal degree in the contraction [14].

\section{Embedding of Lie algebras and the associated contraction}

An embedding of a Lie algebra $\mathfrak{s}^{\prime}$ into a Lie algebra $\mathfrak{s}$ is specified by an isomorphic mapping $f: \mathfrak{s}^{\prime} \longrightarrow \mathfrak{s}$. A special type of embeddings correspond to the so-called regular subalgebras, which can be directly obtained from the Dynkin diagram of semisimple Lie algebras [15]. Each embedding determines an embedding index $j_{f}$ and a branching rule for irreducible representations of $\mathfrak{s}$, which depend essentially on the embedding. For simple complex Lie algebras and maximal semisimple subalgebras, the branching rules have been computed and tabulated up to rank eight [16]. In particular, for the reduction chain $\mathfrak{s}^{\prime} \hookrightarrow_{f} \mathfrak{s}$, the adjoint representation of $\mathfrak{s}$ satisfies the following decomposition

$$
\operatorname{ad} \mathfrak{s}=\operatorname{ad} \mathfrak{s}^{\prime} \oplus R,
$$

where $R$ is a (completely reducible) representation of $\mathfrak{s}^{\prime}$ determined by the embedding index $j_{f} \oint$

In this paragraph we point out that any embedding of (semisimple) Lie algebras $\mathfrak{s}^{\prime} \subset \mathfrak{s}$ naturally induces a contraction of $\mathfrak{s}$ onto an affine Lie algebra. To this extent, consider a basis $\left\{X_{1}, . ., X_{s}, X_{s+1}, . ., X_{n}\right\}$ of $\mathfrak{s}$ such that $\left\{X_{1}, . ., X_{s}\right\}$ is a basis of $\mathfrak{s}^{\prime}$, and $\left\{X_{s+1}, . ., X_{n}\right\}$ spans the representation space of the induced $R$. Over this basis, the structure tensor of $\mathfrak{s}$ can be rewritten as follows

$$
\begin{aligned}
& {\left[X_{i}, X_{j}\right]=\sum_{k=1}^{s} C_{i j}^{k} X_{k}, 1 \leq i, j, k \leq s,} \\
& {\left[X_{i}, X_{j}\right]=\sum_{k=s+1}^{n} C_{i j}^{k} X_{k}, 1 \leq i \leq s, s+1 \leq j, k \leq n,} \\
& {\left[X_{i}, X_{j}\right]=\sum_{k=1}^{s} C_{i j}^{k} X_{k}+\sum_{l=s+1}^{n} C_{i j}^{l} X_{l}, s+1 \leq i, j \leq n .}
\end{aligned}
$$

$\S$ The complete reducibility is actually ensured only if the subalgebra $\mathfrak{s}^{\prime}$ is semisimple. 
For any $t \in \mathbb{R}$ we consider the non-singular linear transformations

$$
\Phi_{t}\left(X_{i}\right)=\left\{\begin{array}{cc}
X_{i}, & 1 \leq i \leq s \\
\frac{1}{t} X_{i}, & s+1 \leq i \leq n
\end{array} .\right.
$$

Expressing the brackets over the transformed basis $\left\{X_{i}^{\prime}=\Phi_{t}\left(X_{i}\right): 1 \leq i \leq n\right\}$ we obtain

$$
\begin{aligned}
& {\left[X_{i}^{\prime}, X_{j}^{\prime}\right]=\sum_{k=1}^{s} C_{i j}^{k} X_{k}^{\prime}, 1 \leq i, j, k \leq s,} \\
& {\left[X_{i}^{\prime}, X_{j}^{\prime}\right]=\sum_{k=s+1}^{n} C_{i j}^{k} X_{k}^{\prime}, 1 \leq i \leq s, s+1 \leq j, k \leq n,} \\
& {\left[X_{i}^{\prime}, X_{j}^{\prime}\right]=\sum_{k=1}^{s} \frac{1}{t^{2}} C_{i j}^{k} X_{k}^{\prime}+\sum_{l=s+1}^{n} \frac{1}{t} C_{i j}^{l} X_{l}^{\prime}, s+1 \leq i, j \leq n .}
\end{aligned}
$$

It follows at once that the subalgebra $\mathfrak{s}^{\prime}$ remains invariant, as well as the representation of $\mathfrak{s}^{\prime}$ over its complementary in $\mathfrak{s}$. These equations also show that the limit

$$
\lim _{t \rightarrow \infty} \Phi_{t}^{-1}\left[\Phi_{t}(X), \Phi_{t}(Y)\right]
$$

exists for any pair of generators $X, Y \in \mathfrak{s}$, we thus obtain a non-trivial contraction $\|$ of $\mathfrak{s}$ denoted by $\mathfrak{g}$ and with non-vanishing brackets

$$
\begin{aligned}
& {\left[X_{i}^{\prime}, X_{j}^{\prime}\right]=\sum_{k=1}^{s} C_{i j}^{k} X_{k}^{\prime}, 1 \leq i, j, k \leq s,} \\
& {\left[X_{i}^{\prime}, X_{j}^{\prime}\right]=\sum_{k=s+1}^{n} C_{i j}^{k} X_{k}^{\prime}, 1 \leq i \leq s, s+1 \leq j, k \leq n .}
\end{aligned}
$$

We observe that if $\mathfrak{s}^{\prime}$ is semisimple, then it coincides with the Levi subalgebra of $\mathfrak{g}$, and the Levi decomposition of this contraction equals

$$
\mathfrak{g}=\mathfrak{s}^{\prime} \vec{\oplus}_{R}(n-s) L_{1},
$$

where $(n-s) L_{1}$ denotes the Abelian algebra of dimension $n-s$. This Lie algebra is affine, and by the contraction we know that $\mathcal{N}(\mathfrak{g}) \geq \mathcal{N}(\mathfrak{s})$. Applying the analytical method, the invariants of $\mathfrak{g}$ are obtained from the solutions of the system:

$$
\begin{aligned}
& \widehat{X}_{i} F=C_{i j}^{k} x_{k} \frac{\partial F}{\partial x_{j}}=0, \quad 1 \leq i \leq s, \\
& \widehat{X}_{s+i} F=C_{s+i, j}^{s+k} x_{s+k} \frac{\partial F}{\partial x_{j}}=0, \quad 1 \leq i, k \leq n-s, 1 \leq j \leq s .
\end{aligned}
$$

Now equation (25) reproduces the subsystem of (44) corresponding to the generators of the embedded subalgebra $\mathfrak{s}^{\prime}$ that must be solved in order to find the missing label operators for the reduction chain $\mathfrak{s}^{\prime} \subset \mathfrak{s}$. This means in particular that any invariant of the contraction $\mathfrak{g}$ is a solution to that system, thus can be taken as candidate for missing label operator, whenever it is functionally independent from the invariants of $\mathfrak{s}$ || This is in fact a simple Inönü-Wigner contraction, following the notation of [11]. 
and $\mathfrak{s}^{\prime}$. As a consequence, we obtain that $\mathcal{N}\left(f\left(\mathfrak{s}^{\prime}\right)\right) \geq \mathcal{N}(\mathfrak{g})$. Combining this inequality with formula (7), we conclude that

$$
\mathcal{N}(f(\mathfrak{s}))=m+\mathcal{N}(\mathfrak{s})+\mathcal{N}\left(\mathfrak{s}^{\prime}\right)-l^{\prime} \geq \mathcal{N}(\mathfrak{g}) \geq \mathcal{N}(\mathfrak{s})
$$

The term $\mathcal{N}(f(\mathfrak{s}))$ on the left hand side gives the total number of available labelling operators, the invariants of $\mathfrak{s}$ and $\mathfrak{s}^{\prime}$ comprised, as shown in [5]. Therefore, if the contraction $\mathfrak{g}$ has enough invariants, we can extract a set of $n$ commuting missing label operators and solve the missing label problem completely. The most important case in physical applications corresponds to reductions chains of the type $\mathfrak{s} \supset \mathfrak{s}^{\prime}$, where $\mathfrak{s}$ is semisimple and $\mathfrak{s}^{\prime}$ is a reductive Lie algebra. Although the contraction method remains completely valid for reductions involving non-reductive algebra-subalgebra chains, in the following we will restrict ourselves to the case of reductive subalgebras, for being the most representative case in Physics.

Suppose therefore that $\mathfrak{s}$ is of $\operatorname{rank} p, \mathfrak{s}^{\prime}$ is a reductive subalgebra and let $\mathfrak{g}=\mathfrak{s}^{\prime} \vec{\oplus}_{R}\left(\operatorname{dim} \mathfrak{s}-\operatorname{dim} \mathfrak{s}^{\prime}\right) k L_{1}$ denote the contraction associated to the chain $\mathfrak{s} \supset \mathfrak{s}^{\prime}$. Let $\left\{C_{1}, . ., C_{p}\right\}$ be the Casimir operators of $\mathfrak{s}$, and $\left\{D_{1}, . ., D_{q}\right\}$ the invariants of $\mathfrak{s}^{\prime}$. Contracting the invariants $C_{i}$ or some appropriate combination of them, we can always obtain $p$ independent invariants of $\mathfrak{g}$. Completing if necessary to a maximal set of invariants of $\mathfrak{g}$, we obtain the fundamental system $\left\{C_{1}^{\prime}, . ., C_{p}^{\prime}, . ., C_{r}^{\prime}\right\}(r \geq p)$. In order to solve the missing label problem using the latter set of functions, the system $\mathcal{F}=\left\{C_{1}^{\prime}, . ., C_{r}^{\prime}\right\}$ must contain at least $n$ functions that are independent on the Casimir invariants of $\mathfrak{s}$ and $\mathfrak{s}^{\prime}$, i.e.,

$$
\operatorname{rank} \mathcal{F}\left(\bmod \left\{C_{1}, . ., C_{p}, D_{1}, . . D_{q}\right\}\right) \geq n .
$$

By the construction, the set $\left\{C_{1}, . ., C_{p}, D_{1}, . ., D_{q-l^{\prime}}\right\}$ is functionally independent. Now the question arises whether adding the invariants of $\mathfrak{g}$ some dependence relations appear. In general, and whenever no invariant is preserved by the contraction, the functions $C_{i}$ and $C_{i}^{\prime}$ are independent. In this case a dependence relation means that some $C_{i}$ is a function of $C_{i}^{\prime}$ and the invariants of $\mathfrak{s}^{\prime}$. We observe that such a dependence relation appears at least for the quadratic Casimir operator $C_{1}$ Indeed, writing $C_{1}$ over the transformed basis (19) we obtain the following decomposition of $C_{1}$ as polynomial in the contraction variable $t$ :

$$
C_{1}=F+t^{2} C_{1}^{\prime}
$$

where $F$ is a quadratic invariant of $\mathfrak{s}^{\prime}$. This decomposition follows from the well known fact that, over the given basis, the quadratic Casimir operator of a reductive subalgebra is always a summand of the quadratic Casimir operator of $\mathfrak{s}+$ As a consequence, we obtain the upper bound

$$
\operatorname{rank}\left\{C_{1}, . ., C_{p}, C_{1}^{\prime}, . ., C_{r}^{\prime}, D_{1}, . ., D_{q}\right\}<\mathcal{N}(\mathfrak{g})+\mathcal{N}(\mathfrak{s})+\mathcal{N}\left(\mathfrak{s}^{\prime}\right)-l^{\prime}
$$

I Is either $\mathfrak{s}$ or $\mathfrak{s}^{\prime}$ is not reductive, this is not applicable, since existence of quadratic operators is not ensured.

+ For higher order invariants, dependence relations could also appear, depending on the homogeneity degree of the invariants of $\mathfrak{s}$ with respect to the generators of the subalgebra. 
Combining the lower and upper bounds (28) and (29) respectively, we obtain a necessary numerical condition on the number of invariants of the contraction $\mathfrak{g}$ :

$$
n<\mathcal{N}(\mathfrak{g}) .
$$

These facts, put together, allow us to characterize when the contraction $\mathfrak{g}$ provides enough labelling operators to solve the missing label problem for $\mathfrak{s} \supset \mathfrak{s}^{\prime}$.

Theorem 1 A necessary and sufficient condition for solving the missing label problem for the reduction $\mathfrak{s} \supset \mathfrak{s}^{\prime}$ by means of the invariants of the associated contraction $\mathfrak{s} \rightsquigarrow \mathfrak{g}=\mathfrak{s}$ is that the affine Lie algebra $\mathfrak{g}$ satisfies the constraints

(i) $\mathcal{N}(\mathfrak{g}) \geq n+1$,

(ii) there are at least $n$ invariants of $\mathfrak{g}$ that are functionally independent from the invariants of $\mathfrak{s}$ and $\mathfrak{s}^{\prime}$.

The first condition, the easiest to evaluate, provides a numerical criterion to decide whether the missing labels can be found by means of the affine algebra $\mathfrak{g}$. Unfortunately, there is no general criterion to decide automatically whether and how many of the contracted invariants are independent on the Casimir operators of $\mathfrak{s}$ and $\mathfrak{s}^{\prime}$. We can however derive the following sufficient condition.

Corollary 1 If the contraction $\mathfrak{g}$ satisfies the numerical condition $\mathcal{N}(\mathfrak{g}) \geq$ $\left\{n+1, \mathcal{N}(\mathfrak{s})+\mathcal{N}\left(\mathfrak{s}^{\prime}\right)+1-l^{\prime}\right\}$, then it solves the $M L P$.

The use of the contraction naturally associated to an embedding has further applications, which can be useful for a general study of affine Lie algebras, in particular inhomogeneous algebras [17, 18, 19]. Let $\mathfrak{s}^{\prime} \hookrightarrow_{f_{1}} \mathfrak{s}$ be an embedding and $\mathfrak{s} \rightsquigarrow \mathfrak{g}=$ $\mathfrak{s}^{\prime} \vec{\oplus}_{R} k L_{1}$ the associated contraction. Since the subalgebra $\mathfrak{s}^{\prime}$ remains invariant by the contraction, we naturally obtain the embedding $f_{2}: \mathfrak{s}^{\prime} \rightarrow \mathfrak{g}$. If we now consider the missing label problem for the latter embedding $*$ we immediately see that the system of PDEs to be solved is exactly the same as for the embedding $f_{1}$. This means that the solutions coincide, and, in particular, their number. This implies that $\mathcal{N}\left(f_{1}\left(\mathfrak{s}^{\prime}\right)\right)=\mathcal{N}\left(f_{2}\left(\mathfrak{s}^{\prime}\right)\right)$. Recall that for each embedding the number of independent solutions is given by

$$
\begin{aligned}
& \mathcal{N}\left(f_{1}\left(\mathfrak{s}^{\prime}\right)\right)=\operatorname{dim} \mathfrak{s}-\operatorname{dim} \mathfrak{s}^{\prime}+l^{\prime}, \\
& \mathcal{N}\left(f_{2}\left(\mathfrak{s}^{\prime}\right)\right)=\operatorname{dim} \mathfrak{g}-\operatorname{dim} \mathfrak{s}^{\prime}+l_{1}^{\prime},
\end{aligned}
$$

where $l_{1}^{\prime}$ denotes the number of common invariants of $\mathfrak{s}^{\prime}$ and $\mathfrak{g}$. Since contractions preserve the dimension, we conclude from formula (31) that $l^{\prime}=l_{1}^{\prime}$, that is, the subalgebra $\mathfrak{s}^{\prime}$ has the same number of common invariants with $\mathfrak{s}$ than with the contraction $\mathfrak{g}$. On the other hand, using the reformulation (7)

$$
\begin{aligned}
& \mathcal{N}\left(f_{1}\left(\mathfrak{s}^{\prime}\right)\right)=m+\mathcal{N}(\mathfrak{s})+\mathcal{N}\left(\mathfrak{s}^{\prime}\right)-l^{\prime} \\
& \mathcal{N}\left(f_{2}\left(\mathfrak{s}^{\prime}\right)\right)=\widetilde{m}+\mathcal{N}(\mathfrak{g})+\mathcal{N}\left(\mathfrak{s}^{\prime}\right)-l_{1}^{\prime}
\end{aligned}
$$

\footnotetext{
* Actually the mappings $f_{1}$ and $f_{2}$ are the same, but we distinguish the target algebra by the indices.
} 
we deduce that

$$
m-\widetilde{m}=\mathcal{N}(\mathfrak{g})-\mathcal{N}(s) \geq 0 .
$$

This result tells us that the number of available labelling operators for the reduction chain $\mathfrak{s} \supset \mathfrak{s}^{\prime}$ is always higher than that of the chain $\mathfrak{g} \supset \mathfrak{s}^{\prime}$. Even more, the inequality (33) gives us a criterion to compute the number of invariants of contractions in dependence of the available missing label operators with respect to an invariant subalgebra.

Proposition 1 Let $\mathfrak{s} \rightsquigarrow \mathfrak{g}$ be a contraction such that the subalgebra $\mathfrak{s}^{\prime}$ is (maximal) invariant. Then following equality holds:

$$
\mathcal{N}(\mathfrak{g})=\mathcal{N}(s)+m-\widetilde{m},
$$

where $m$ and $\widetilde{m}$ is the number of available missing label operators for the algebra subalgebra chain $\mathfrak{s} \supset \mathfrak{s}^{\prime}$ and $\mathfrak{g} \supset \mathfrak{s}^{\prime}$, respectively.

This result has useful applications, like the determination of the number of invariants of some inhomogeneous Lie algebras. As a particular case, we obtain the following upper bound

$$
\mathcal{N}(\mathfrak{g}) \leq \mathcal{N}(s)+m .
$$

This bound has an important interpretation, namely, that the number of invariants of a contraction is, in some sense, determined by the number of available missing label operators for the missing label problem with respect to a maximal subalgebra of $\mathfrak{s}$ that remains invariant by the contraction. This fact establishes a quite strong restriction to semidirect products of semisimple and Abelian Lie algebras to appear as contractions of semisimple Lie algebras [20].

\section{The case $n=m=0$}

In the case of zero missing labels, the invariants of the algebra-subalgebra chain provide a complete description of the states. This situation is not uncommon for certain canonical embeddings, such as the inclusions $\mathfrak{s o}(N) \subset \mathfrak{s o}(N+1)$ of (pseudo)-orthogonal Lie algebras. Even if this case is trivial, its interpretation in terms of the associated contraction provides some interesting information concerning the invariants of the contraction.

At first, if $m=0$, then by formula (33) we have $\mathcal{N}(\mathfrak{g})=\mathcal{N}(s)$, i.e., the contraction determined by the embedding $\mathfrak{s} \supset \mathfrak{s}^{\prime}$ preserves the number of invariants. It is worth to be observed that the converse does not necessarily hold. Moreover, by formula (2), we have

$$
0=m=\operatorname{dim} \mathfrak{s}-\operatorname{dim} \mathfrak{s}^{\prime}-\mathcal{N}(\mathfrak{s})-\mathcal{N}\left(\mathfrak{s}^{\prime}\right)+2 l^{\prime} .
$$

In absence of additional internal labels, the system $\widehat{X}_{i} F=0$ for the generators of $\mathfrak{s}^{\prime}$ has exactly

$$
\mathcal{N}(f(\mathfrak{s}))=\mathcal{N}(\mathfrak{s})+\mathcal{N}\left(\mathfrak{s}^{\prime}\right)-l^{\prime}
$$


solutions. Since any invariant of the contraction $\mathfrak{g}=\mathfrak{s}^{\prime} \vec{\oplus}_{R}\left(\operatorname{dim} \mathfrak{s}-\operatorname{dim} \mathfrak{s}^{\prime}\right) L_{1}$ is a special solution of this system, the latter equation tells that any invariant of $\mathfrak{g}$ is functionally dependent on the invariants of $\mathfrak{s}$ and the subalgebra $\mathfrak{s}^{\prime}$. That is, the Casimir invariants of the algebra-subalgebra chain completely determine the invariants of the contraction $\sharp$ Expressed in another way, in this situation, polynomial functions of the invariants of $\mathfrak{s}$ and the contraction $\mathfrak{g}$ allow to recover naturally the invariants of the subalgebra.

These observations provide a new (and very short) proof of the fact that the number of invariants for inhomogeneous pseudo-orthogonal Lie algebras is given by

$$
\mathcal{N}(I \mathfrak{s o}(p, q))=\left[\frac{p+q+1}{2}\right] .
$$

In fact, it is straightforward to verify that $n=0$, and since $I \mathfrak{s o}(p, q)$ is a contraction of $\mathfrak{s o}(p+1, q)$, the result follows at once from formula (34). Moreover, the invariants of $I \mathfrak{s o}(p, q))$ can be obtained from the invariants of $\mathfrak{s o}(p+1, q)$ and $\mathfrak{s o}(p, q)$. This explains in some manner why the classical Gel'fand method applies so well to inhomogeneous algebras of this kind [19].

As example, consider the embedding $\mathfrak{s o}(3,1) \hookrightarrow \mathfrak{s o}(4,1)$ of the Lorentz algebra into the Anti De Sitter algebra $\mathfrak{s o}(4,1)$. Using the kinematical basis $\left\{J_{\alpha}, P_{\alpha}, K_{\alpha}, H\right\}_{1 \leq \alpha \leq 3}$, where $J_{\alpha}$ are spatial rotations, $P_{\alpha}$ spatial translations, $K_{\alpha}$ the boosts and $H$ the time translation, the non-trivial brackets of $\mathfrak{s o}(4,1)$ are

$$
\begin{aligned}
& {\left[J_{\alpha}, J_{\beta}\right]=\varepsilon^{\alpha \beta \gamma} J_{\gamma}, \quad\left[J_{\alpha}, P_{\beta}\right]=\varepsilon^{\alpha \beta \gamma} P_{\gamma}, \quad\left[J_{\alpha}, K_{\beta}\right]=\varepsilon^{\alpha \beta \gamma} K_{\gamma}, \quad\left[H, P_{\alpha}\right]=\varepsilon^{\alpha \beta \gamma} K_{\alpha},} \\
& {\left[H, K_{\alpha}\right]=\varepsilon^{\alpha \beta \gamma} P_{\alpha}, \quad\left[P_{\alpha}, P_{\beta}\right]=\varepsilon^{\alpha \beta \gamma} J_{\gamma}, \quad\left[K_{\alpha}, K_{\beta}\right]=-\varepsilon^{\alpha \beta \gamma} J_{\gamma}, \quad\left[P_{\alpha}, K_{\alpha}\right]=H .}
\end{aligned}
$$

It follows at once that $\mathfrak{s o}(3,1)$ is generated by the rotations and boosts. In this case there are no missing labels, thus $n=m=0$. The corresponding contraction defined by the linear maps

$$
J_{\alpha}^{\prime}=J_{\alpha}, P_{\alpha}^{\prime}=\frac{1}{t} P_{\alpha}, K_{\alpha}^{\prime}=K_{\alpha}, H^{\prime}=\frac{1}{t} H
$$

leads to the Poincaré algebra $I \mathfrak{s o}(3,1)$. Over this basis, the Casimir operators of $\mathfrak{s o}(4,1)$ are

$C_{2}=j_{\alpha} j^{\alpha}+p_{\alpha} p^{\alpha}-k_{\alpha} k^{\alpha}-h^{2}$

$C_{4}=j_{\alpha} j^{\alpha} h^{2}+\left(p_{\alpha} p^{\alpha}\right)\left(k_{\alpha} k^{\alpha}\right)-\left(p_{\alpha} k^{\alpha}\right)^{2}+\left(p_{\alpha} j^{\alpha}\right)^{2}-\left(j_{\alpha} k^{\alpha}\right)^{2}-2 \varepsilon^{\alpha \beta \gamma} j_{\alpha} p_{\beta} k_{\gamma} h$.

Contraction of these invariants give the Casimir operators of the Poincaré algebra

$$
\begin{aligned}
& C_{2}^{\prime}=p_{\alpha} p^{\alpha}-h^{2} \\
& C_{4}^{\prime}=j_{\alpha} j^{\alpha} h^{2}+\left(p_{\alpha} p^{\alpha}\right)\left(k_{\alpha} k^{\alpha}\right)-\left(p_{\alpha} k^{\alpha}\right)^{2}+\left(p_{\alpha} j^{\alpha}\right)^{2}-2 \varepsilon^{\alpha \beta \gamma} j_{\alpha} p_{\beta} k_{\gamma} h .
\end{aligned}
$$

Now observe that $C_{21}=p_{\alpha} p^{\alpha}-k_{\alpha} k^{\alpha}$ and $C_{22}=j_{a} k^{\alpha}$ are the Casimir operators of the $\mathfrak{s o}(3,1)$ subalgebra. It follows that

$$
C_{2}=C_{2}^{\prime}+C_{21}, C_{4}=C_{4}^{\prime}-C_{22}^{2},
$$

$\sharp$ Of course, if $\mathcal{N}\left(\mathfrak{s}^{\prime}\right)=0$, this assertion fails, but for reductive subalgebras this situation is excluded. 
i.e., the mass squared and spin squared operators of the Poincaré algebra are obtainable as a difference of the Casimir operators of the Lorentz and De Sitter Lie algebras, and therefore the information they provide is already contained in the reduction chain.

\section{The case $n=1, m=2$}

In the case of one missing label operator, any solution of the contraction $\mathfrak{g}$ that is independent of the invariants of the algebra-subalgebra chain can be used. No commutation problems arise at this step. Formula (34) establishes the maximal possible number for the invariants of $\mathfrak{g}$ :

$$
\mathcal{N}(\mathfrak{g}) \leq \mathcal{N}(s)+2
$$

For the case of semisimple Lie algebra $\mathfrak{s}$ and maximal reductive subalgebra $\mathfrak{s}^{\prime}$, there are eight cases with one missing label [5, 21]. Most of these chains have been solved finding finite integrity bases, that is, a set of elementary subgroup scalar such that any other can be expressed by a polynomial in them. All eight cases can also be solved applying the contraction method. In order to illustrate how the contraction method works, we consider two representative cases, and resume the results for the remaining cases in Table 1.

\subsection{The $\mathfrak{s u}(3) \supset \mathfrak{s o}(3)$ reduction}

This reduction chain, first considered in atomic physics by Elliott, is probably the best known and best studied case concerning the missing label problem. A complete set of commuting operators and their eigenvalues for different irreducible representations of $\mathfrak{s u}(3)$ were first determined in [22].

The $\mathfrak{s o}(3)$ subalgebra is naturally identified with the three orbital angular momentum operators, while the remaining five generators transform under rotations like the elements of a second rank tensor [1, 3]. Here we consider a basis $\left\{L_{i}, T_{j k}\right\}$ formed by rotations $L_{i}$ and the operators $T_{i k}$ and commutation relations

$$
\begin{aligned}
& {\left[L_{j}, L_{k}\right]=i \varepsilon_{j k l} L_{l}, \quad\left[L_{j}, T_{k l}\right]=i \varepsilon_{j k m} T_{l m}+i \varepsilon_{j l m} T_{k m},} \\
& {\left[T_{j k}, T_{l m}\right]=\frac{i}{4}\left\{\delta_{j}^{l} \varepsilon_{k m n}+\delta_{j}^{m} \varepsilon_{k \ln }+\delta_{k}^{l} \varepsilon_{j m n}+\delta_{k}^{m} \varepsilon_{j l n}\right\} L_{n},}
\end{aligned}
$$

where $T_{33}+\left(T_{11}+T_{22}\right)=0$. The symmetrized Casimir operators, following the notation of [22], are given by $C^{(2)}=L_{i} L_{i}+2 T_{i k} T_{i k}, \quad C^{(3)}=L_{i} T_{i k} L_{k}-\frac{4}{3} T_{i k} T_{k l} T_{l i}$ and $C^{(2,0)}=L_{i} L_{i}$. The contraction $\mathfrak{g}$ associated to this reduction has Levi decomposition $\mathfrak{g}=\mathfrak{s o}(3) \vec{\oplus}_{R_{5}^{I}} 5 L_{1}$, where $R_{5}^{I}$ denotes the five dimensional irreducible representation of $\mathfrak{s o}(3)$. This is equivalent to the rotor algebra $\left[\mathbb{R}^{5}\right] S O(3)$ studied in [2]. It is straightforward to verify that $\mathcal{N}(\mathfrak{g})=2$. Therefore, a basis of invariants of $\mathfrak{g}$ can be obtained by contraction of $C^{(2)}$ and $C^{(3)}$. Specifically, we get the (unsymmetrized) Casimir invariants

$$
\begin{aligned}
& C_{2}=2 t_{i k} t^{i k}, \\
& C_{3}=t_{i k} t^{k l} t_{l i} .
\end{aligned}
$$


As already observed, $C_{2}$ is functionally dependent on $C^{(2)}$ and $C^{(2,0)}$, therefore of no use for the MLP. The independence of $\left\{C^{(2)}, C^{(3)}, C^{(2,0)}, C_{3}\right\}$ follows from the Jacobian

$$
\frac{\partial\left\{C^{(2)}, C^{(3)}, C^{(2,0)}, C_{3}\right\}}{\partial\left\{l_{2}, l_{3}, t_{11}, t_{12}\right\}} \neq 0 .
$$

The invariant $C_{3}$ is therefore sufficient to solve the missing label problem. In fact, we can recover the missing label operator $X^{(3)}$ from [22] by simply considering the linear combination

$$
X^{(3)}=C^{(3)}+\frac{4}{3}\left\{C_{3}\right\}_{\text {symmetrized }} .
$$

This operator is equivalent to the third order operator obtained by Bargmann and Moshinsky in [23], and also to the operator determined in [2] using the K-matrix approach. It is observed that the fourth order operator $X^{(4)}=L_{i} T_{i j} T_{j k} L_{k}$ cannot be obtained from the invariants of $\mathfrak{s u}(3), \mathfrak{s o}(3)$ and the contraction $\mathfrak{g}$. This is essentially due to the fact that the fundamental Casimir operators of $\mathfrak{s u}(3)$ have degree two and three.

\subsection{The seniority model}

The reduction $\mathfrak{s o}(5) \supset \mathfrak{s u}(2) \times \mathfrak{u}(1)$ has been used in the treatment of the paring force between particles in the same nuclear shell, and is usually referred to as the seniority model [24].

In order to analyze this chain, we use the same basis $\left\{U_{ \pm}, U_{3}, V_{3}, V_{ \pm}, S_{ \pm}, T_{ \pm}\right\}$of [25]. The $\mathfrak{s u}(2) \times \mathfrak{u}(1)$ subalgebra is generated by the operators $\left\{U_{ \pm}, U_{3}, V_{3}\right\}$. The nonzero brackets are given by

$$
\begin{array}{llll}
{\left[U_{ \pm}, U_{3}\right]=\mp U_{ \pm},} & {\left[U_{+}, U_{-}\right]=2 U_{3},} & {\left[U_{ \pm}, V_{ \pm}\right]=\mp 2 S_{ \pm},} & {\left[U_{ \pm}, V_{\mp}\right]=\mp 2 T_{ \pm},} \\
{\left[U_{ \pm}, S_{\mp}\right]= \pm V_{\mp},} & {\left[U_{ \pm}, T_{\mp}\right]= \pm V_{\mp},} & {\left[U_{3}, S_{ \pm}\right]= \pm S_{ \pm},} & {\left[U_{3}, T_{ \pm}\right]= \pm T_{ \pm},} \\
{\left[V_{3}, S_{ \pm}\right]= \pm S_{ \pm},} & {\left[V_{3}, T_{ \pm}\right]=\mp T_{ \pm},} & {\left[V_{+}, V_{-}\right]=2 V_{3},} & {\left[V_{ \pm}, V_{3}\right]=\mp V_{ \pm},} \\
{\left[V_{ \pm}, S_{\mp}\right]=\mp U_{\mp},} & {\left[V_{ \pm}, T_{ \pm}\right]= \pm U_{ \pm},} & {\left[S_{+}, S_{-}\right]=U_{3}+V_{3},} & {\left[T_{+}, T_{-}\right]=U_{3}-V_{3} .}
\end{array}
$$

Over this basis, the (unsymmetrized) Casimir operators of $\mathfrak{s o}(5)$ can be chosen as

$$
\begin{aligned}
& C_{2}=u_{+} u_{-}+u_{3}^{2}+v_{3}^{2}+v_{+} v_{-}+2\left(s_{+} s_{-}+t_{+} t_{-}\right) \\
& C_{4}=\left(u_{+} u_{-}+u_{3}^{2}\right) v_{3}^{2}+u_{+} u_{-}\left(s_{+} s_{-}+t_{+} t_{-}\right)+u_{+}^{2} s_{-} t_{-}+u_{-}^{2} s_{+} t_{+}+2 u_{3} v_{3}\left(s_{+} s_{-}-t_{+} t_{-}\right) \\
& +\left(\left(t_{-} v_{-}-s_{-} v_{+}\right) u_{+}+\left(t_{+} v_{+}-s_{+} v_{-}\right) u_{-}\right) v_{3}+\left(\left(t_{+} v_{+}+s_{+} v_{-}\right) u_{-}+\left(s_{-} v_{+}+t_{-} v_{-}\right) u_{+}\right) u_{3} \\
& +v_{+} v_{-} s_{+} s_{-}+u_{3}^{2} v_{+} v_{-}+\left(s_{+} s_{-}-t_{+} t_{-}\right)^{2}-v_{+}^{2} s_{-} t_{+}-v_{-}^{2} s_{+} t_{-}+v_{+} v_{-} t_{+} t_{-},
\end{aligned}
$$

while those of the subalgebra are given by $C_{21}=u_{+} u_{-}+u_{3}^{2}, C_{22}=v_{3}$. The associated contraction $\mathfrak{g}$ is easily seen to have exactly two invariants, which can be obtained from those of $\mathfrak{s o}(5)$ by the contraction method:

$$
\begin{aligned}
& C_{2}^{\prime}=v_{+} v_{-}+2\left(s_{+} s_{-}+t_{+} t_{-}\right) \\
& C_{4}^{\prime}=v_{+} v_{-} s_{+} s_{-}+\left(s_{+} s_{-}-t_{+} t_{-}\right)^{2}-v_{+}^{2} s_{-} t_{+}-v_{-}^{2} s_{+} t_{-}+v_{+} v_{-} t_{+} t_{-} .
\end{aligned}
$$


Table 1. Comparison of missing labels of [5] and those obtained by contraction.

\begin{tabular}{l|ccccc}
$\mathfrak{s} \supset \mathfrak{s}^{\prime}$ & $\mathcal{N}(\mathfrak{g})$ & $\mathcal{N}\left(f\left(\mathfrak{s}^{\prime}\right)\right)$ & $\operatorname{rank} \mathcal{F}$ & Order of $\Phi$ & Operator of [5] \\
\hline $\mathfrak{s u}(3) \supset \mathfrak{s o}(3)$ & 2 & 5 & 4 & 3 & $X^{(3) \text { a }}$ \\
$\mathfrak{s o}(5) \supset \mathfrak{s u}(2) \times \mathfrak{u}(1)$ & 2 & 6 & 5 & 4 & $U V L^{2}$ \\
$G_{2} \supset \mathfrak{s u}(3)$ & 2 & 5 & 4 & 6 & $U^{3} V^{3}$ \\
$\mathfrak{s p}(6) \supset \mathfrak{s p}(4) \times \mathfrak{s u}(2)$ & 3 & 8 & 7 & 6 & $Q^{3} T^{2} L$ \\
$\mathfrak{s o}(7) \supset G_{2}$ & 3 & 7 & 6 & 6 & $T^{4} S^{2}$ \\
$\mathfrak{s u}(4) \supset[\mathfrak{s u}(2)]^{2} \times \mathfrak{u}(1)$ & 3 & 7 & 6 & 4 & $U V S T$ \\
$\mathfrak{s u}(3) \times \mathfrak{s u}(3) \supset \mathfrak{s u}(3)$ & 2 & 8 & 7 & 3 & $U V^{2}$ \\
{$[\mathfrak{s u}(2)]^{3} \supset \mathfrak{s u}(2)$} & 3 & 6 & 5 & 2 & $-{ }^{\mathrm{b}}$ \\
\hline \hline
\end{tabular}

a The notation for the operator corresponds to that used in [22].

b This case, omitted in [5], was first considered in [21].

As expected, we have $C_{2}=C_{2}^{\prime}+C_{21}+C_{22}^{2}$, thus at most $C_{4}^{\prime}$ is independent on the invariants of $\mathfrak{s o}(5)$ and $\mathfrak{s u}(2) \times \mathfrak{u}(1)$. A short computation shows that

$$
\operatorname{rank}\left\{C_{2}, C_{4}, C_{21}, C_{22}, C_{4}^{\prime}\right\}=5,
$$

showing that the missing label problem can be solved using the contraction $\mathfrak{g}$. Now, after some manipulation we can arrive at the expression $\Omega_{4}=C_{4}-C_{4}^{\prime}-C_{21} C_{22}^{2}$ explicitly given by

$\Omega_{4}=u_{+} u_{-}\left(s_{+} s_{-}+t_{+} t_{-}\right)+u_{3}^{2} v_{+} v_{-}+u_{+}^{2} s_{-} t_{-}+u_{-}^{2} s_{+} t_{+}+2 u_{3} v_{3}\left(s_{+} s_{-}-t_{+} t_{-}\right)$ $+\left(\left(t_{-} v_{-}-s_{-} v_{+}\right) u_{+}+\left(t_{+} v_{+}-s_{+} v_{-}\right) u_{-}\right) v_{3}+\left(\left(t_{+} v_{+}+s_{+} v_{-}\right) u_{-}+\left(s_{-} v_{+}+t_{-} v_{-}\right) u_{+}\right) u_{3}$.

This operator is obviously independent on the invariants of the orthogonal algebra and the subalgebra, and can therefore be taken as the missing operator. It can be verified that $\Omega_{4}$, after symmetrization, coincides with the fourth order operator $U V L^{2}$ found in [5]. The remaining third order operator cannot be obtained using the contraction $\mathfrak{g}$. In this case, this is a consequence of the non-existence of cubic Casimir operators for the orthogonal algebra $\mathfrak{s o}(5)$.

\section{The case $n=2, m=4$}

The case with two missing labels is notably more complicated, because in addition to determine two missing label operators, these must commute. Although a considerable number of cases has been studied, only for a few the most general form of missing label operators has been analyzed in detail, such as the Wigner supermultiplet $\mathfrak{s u}(4) \supset$ $\mathfrak{s u}(2) \times \mathfrak{s u}(2)$ [26, 27] or the chain $\mathfrak{s o}(5) \supset \mathfrak{s u}(2)$ used for the classification of nuclear surfon states [24].

\subsection{The supermultiplet model}

This model, used by Wigner to describe light nuclei, has been considered in detail by various authors, usually by means of enveloping algebras [26, 27, 28]. It has been shown that the set of available operators is partitioned into two separate sets, the 
Moshinky-Nagel operators $\Omega, \Phi$ and two other operators $O_{1}, O_{2}$, first found in [26] and later evaluated numerically in [27]. We start from the same basis $\left\{S_{i}, T_{j}, Q_{\alpha \beta}\right\}$ used in [28], where $1 \leq i, j, \alpha, \beta \leq 3$. The non-vanishing brackets of $\mathfrak{s u}(4)$ are

$$
\begin{aligned}
& {\left[S_{i}, S_{j}\right]=i \varepsilon_{i j k} S_{k},\left[T_{i}, T_{j}\right]=i \varepsilon_{i j k} T_{k},\left[S_{i}, Q_{j \alpha}\right]=i \varepsilon_{i j k} Q_{k \alpha},\left[T_{\alpha}, Q_{i \beta}\right]=i \varepsilon_{\alpha \beta \gamma} Q_{i \gamma},} \\
& {\left[Q_{i \alpha}, Q_{j \beta}\right]=\frac{i}{4}\left\{\delta_{\alpha \beta} \varepsilon_{i j k} S_{k}+\delta_{i j} \varepsilon_{\alpha \beta \gamma} T_{\gamma}\right\},}
\end{aligned}
$$

where $\varepsilon_{i j k}$ is the completely antisymmetric tensor. The $\mathfrak{s u}(2) \times \mathfrak{s u}(2)$-subalgebra is generated by the operators $\left\{S_{i}, T_{j}\right\}$. It follows easily from the brackets that the generators of $\mathfrak{s u}(4)$ decompose as the following $\mathfrak{s u}(2) \times \mathfrak{s u}(2)$-representation

$$
R=\left(D_{1} \otimes D_{0}\right) \oplus\left(D_{0} \otimes D_{1}\right) \oplus\left(D_{1} \otimes D_{1}\right),
$$

where $D_{1}$ denotes the adjoint representation of $\mathfrak{s u}(2)$ and $D_{0}$ the trivial representation. The two missing label operators are therefore determined by the system of differential equations

$\widehat{S}_{i} F=\epsilon_{i j k} s_{k} \frac{\partial F}{\partial s_{j}}+\epsilon_{i j k} q_{k l} \frac{\partial F}{\partial q_{k l}}=0, \quad \widehat{T}_{\alpha} F=\epsilon_{\alpha \beta \gamma} t_{\gamma} \frac{\partial F}{\partial t_{\beta}}+\epsilon_{\beta \gamma \mu} q_{\alpha \mu} \frac{\partial F}{\partial q_{\beta \mu}}=0, \quad i=1,2,3$

corresponding to the generators of the subalgebra. From the nine independent solutions, five of them correspond to invariants of $\mathfrak{s u}(4)$ and the subalgebra. The Casimir operators can be taken as.

$$
\begin{aligned}
& C_{2}=s_{\alpha} s^{\alpha}+t_{\beta} t^{\beta}+4 q_{\alpha \beta} q^{\alpha \beta} \\
& C_{3}=s_{\alpha} t_{\beta} q^{\alpha \beta}-4 \varepsilon^{i j k} \varepsilon^{\alpha \beta \gamma} q_{i \alpha} q_{j \beta} q_{k \gamma} \\
& C_{4}=16\left\{\varepsilon_{\alpha \beta \gamma}^{2}\left(q_{\alpha \beta}^{2}\left(q_{\alpha \gamma}^{2}+q_{\gamma \beta}^{2}\right)+2 q_{\alpha \alpha}^{2}\left(q_{\alpha \gamma}^{2}+q_{\beta \alpha}^{2}\right)-2 q_{\alpha \alpha} q_{\alpha \beta} q_{\gamma \alpha} q_{\gamma \beta}+3 q_{\alpha \beta}^{2}\left(q_{\gamma \alpha}^{2}+q_{\gamma \gamma}^{2}\right)\right)\right. \\
& \left.+\sum_{a<\beta}\left(3\left(q_{\alpha \alpha}^{2} q_{\beta \beta}^{2}+q_{\alpha \beta}^{2} q_{\beta \alpha}^{2}\right)-2 q_{\alpha \alpha} q_{\beta \beta} q_{\alpha \beta} q_{\beta \alpha}\right)+q_{\alpha \beta}^{4}\right\}+\left(s_{\alpha} s^{\alpha}\right)^{2}+\left(t_{\beta} t^{\beta}\right)^{2}+3 s_{\alpha} s^{\alpha} t_{\beta} t^{\beta} \\
& +2^{3} q_{\alpha \beta}^{2}\left(s_{\alpha} s^{\alpha}+t_{\beta} t^{\beta}\right)+4\left\{t_{\alpha} t_{\beta} q_{\gamma \alpha} q_{\gamma \beta}+s_{\alpha} s_{\beta} q_{\alpha \gamma} q_{\beta \gamma}-\varepsilon_{\alpha \beta \gamma} \varepsilon_{\mu \nu \rho} s_{\mu} t_{\alpha} q_{\nu \beta} q_{\rho \gamma}\right\}
\end{aligned}
$$

for $\mathfrak{s u}(4)$, and $C_{21}=s_{\alpha} s^{\alpha}, \quad C_{22}=t_{\beta} t^{\beta}$ for the subalgebra. In this case, the contraction $\mathfrak{g}=(\mathfrak{s u}(2) \times \mathfrak{s u}(2)) \vec{\oplus}_{D_{1} \otimes D_{1}} 9 L_{1}$ associated to the embedding has the following non-trivial brackets

$$
\left[S_{i}, S_{j}\right]=i \varepsilon_{i j k} S_{k},\left[T_{i}, T_{j}\right]=i \varepsilon_{i j k} T_{k},\left[S_{i}, Q_{j \alpha}\right]=i \varepsilon_{i j k} Q_{k \alpha},\left[T_{\alpha}, Q_{i \beta}\right]=i \varepsilon_{\alpha \beta \gamma} Q_{i \gamma} .
$$

Using formula (6) we easily get $\mathcal{N}(\mathfrak{g})=3$. Contracting the invariants we obtain three independent invariants of $\mathfrak{g}$, given respectively by

$$
\begin{aligned}
& C_{2}^{\prime}=4 q_{\alpha \beta} q^{\alpha \beta} \\
& C_{3}^{\prime}=-4 \varepsilon^{i j k} \varepsilon^{\alpha \beta \gamma} q_{i \alpha} q_{j \beta} q_{k \gamma} \\
& C_{4}^{\prime}=16\left\{\varepsilon_{\alpha \beta \gamma}^{2}\left(q_{\alpha \beta}^{2}\left(q_{\alpha \gamma}^{2}+q_{\gamma \beta}^{2}\right)+2 q_{\alpha \alpha}^{2}\left(q_{\alpha \gamma}^{2}+q_{\beta \alpha}^{2}\right)-2 q_{\alpha \alpha} q_{\alpha \beta} q_{\gamma \alpha} q_{\gamma \beta}+3 q_{\alpha \beta}^{2}\left(q_{\gamma \alpha}^{2}+q_{\gamma \gamma}^{2}\right)\right)\right. \\
& \left.+q_{\alpha \beta}^{4}+\sum_{a<\beta}\left(3\left(q_{\alpha \alpha}^{2} q_{\beta \beta}^{2}+q_{\alpha \beta}^{2} q_{\beta \alpha}^{2}\right)-2 q_{\alpha \alpha} q_{\beta \beta} q_{\alpha \beta} q_{\beta \alpha}\right)\right\}
\end{aligned}
$$

As observed, the quadratic Casimir operator of $\mathfrak{g}$ satisfies the condition $C_{2}-C_{2}^{\prime}=$ $C_{21}+C_{22}$, and is therefore dependent. To prove that $\mathcal{F}=\left\{C_{2}, C_{3}, C_{4}, C_{21}, C_{22}, C_{3}^{\prime}, C_{4}^{\prime}\right\}$ 
is a functionally independent set, we consider the Jacobian with respect to the variables $\left\{s_{2}, s_{3}, t_{1}, t_{2}, q_{11}, q_{12}, q_{23}\right\}$ :

$$
\frac{\partial\left(C_{21}, C_{2}, C_{3}, C_{4}, C_{2}^{\prime}, C_{3}^{\prime}, C_{4}^{\prime}\right)}{\partial\left(s_{2}, s_{3}, t_{1}, t_{2}, q_{11}, q_{12}, q_{23}\right)} \neq 0 .
$$

Actually, this is a maximal set of independent functions among the invariants of the intervening Lie algebras $\mathfrak{s u}(4), \mathfrak{s u}(2) \times \mathfrak{s u}(2)$ and $\mathfrak{g}$. This means that the contraction method provides at most two of the four available operators. If we take the difference of the cubic invariants of $\mathfrak{s u}(4)$ and $\mathfrak{g}$, we recover exactly the cubic operator $\Omega$ of Moshinsky and Nagel [28]:

$$
C_{3}-C_{3}^{\prime}=\Omega=s_{\alpha} t_{\beta} q^{\alpha \beta} .
$$

As known, the operator $\Omega$ only commutes with the fourth order operator $\Phi$ defined by

$$
\Phi=S_{i} S_{j} Q_{i \alpha} Q_{j \alpha}+Q_{i \alpha} Q_{i \beta} T_{\alpha} T_{\beta}-\epsilon_{i j k} \epsilon_{\alpha \beta \gamma} S_{i} T_{\alpha} Q_{j \beta} Q_{k \gamma} .
$$

With some more effort we can express $\Phi$ with the help of the preceding functions of $\mathcal{F}$, obtaining

$$
\Phi=\frac{1}{4}\left\{C_{4}-C_{4}^{\prime}+C_{21}^{2}-C_{2}^{2}+C_{2}^{\prime 2}-C_{21}\left(C_{2}^{\prime}-C_{2}\right)\right\} .
$$

This means that the commuting $\Omega-\Phi$ operators of Moshinky-Nagel are completely determined by the contraction associated to the embedding of spin-isospin subalgebra in $\mathfrak{s u}(4)$, while the other pair of commuting operators, being summands of $\Phi$, cannot be obtained by this method.

\subsection{The nuclear surfon model}

The reduction chain $\mathfrak{s o}(5) \supset \mathfrak{s u}(2)$ has been analyzed in [29], where two commuting missing label operators of degrees four and six were found. The authors looked for the simplest possible operators solving the labelling problem. We reconsider the problem with the contraction method. As in [29], we choose the basis of $\mathfrak{s o}(5)$ to consist of generators $\left\{L_{0}, L_{1}, L_{-1}\right\}$ with brackets $\left[L_{0}, L_{ \pm 1}\right]= \pm L_{ \pm 1},\left[L_{1}, L_{-1}\right]=2 L_{0}$ together with an irreducible tensor representation $Q_{\mu}(\mu=-3.3)$. The brackets of $\mathfrak{s o}(5)$ over this basis are given in Table 2. According to [29], the Casimir operators of $\mathfrak{s o}(3)$ and $\mathfrak{s o}(5)$ are given respectively by:

$$
\begin{aligned}
& C_{21}=l_{0}^{2}+l_{1} l_{-1}, \\
& C_{2}=l_{0}^{2}+l_{1} l_{-1}-\frac{2}{5}\left(q_{3} q_{-3}+q_{1} q_{-1}\right)+\frac{1}{15} q_{2} q_{2}+q_{0}^{2}, \\
& C_{4}=l_{0}^{3} q_{0}+\frac{1}{6}\left(l_{-1} q_{1}-l_{1} q_{-1}+\frac{1}{2} l_{1} l_{-1}\right) q_{0}^{2}+\frac{1}{6}\left(q_{3} q_{-1} q_{-2}+q_{2} q_{1} q_{-3}+\frac{1}{3} q_{1}^{2} q_{-2}+\frac{1}{3} q_{2} q_{-1}^{2}\right) q_{0}+ \\
& -\frac{1}{3}\left(\frac{1}{3} l_{-1} q_{-1}+\frac{1}{2} l_{0} q_{-2}+\frac{2}{3} l_{1} q_{-3}\right) q_{1}^{2}+\frac{1}{3}\left(\frac{2}{3} l_{-1} q_{3}+\frac{1}{3} l_{1} q_{1}-\frac{1}{2} l_{0} q_{2}\right) q_{-1}^{2}+\frac{1}{4}\left(l_{1}^{3} q_{-3}-l_{-1}^{3} q_{3}\right) \\
& \frac{1}{3}\left(\frac{1}{20} q_{2} q_{-2}-q_{1} q_{-1}-3 l_{-1} q_{1}+\frac{7}{4} q_{0}^{2}+3 l_{1} q_{-1}+\frac{1}{5} q_{3} q_{-3}\right) l_{0}^{2}-\frac{3}{100} q_{3}^{2} q_{-3}^{2}-\frac{q_{-2} q_{2}}{540}\left(q_{1} q_{-1}+36 q_{0}^{2}\right)
\end{aligned}
$$




$$
\begin{aligned}
& +\frac{1}{12}\left(q_{-1}^{2}-3 l_{-1} q_{-1}+3 l_{0} q_{-2}+q_{1} q_{-3}-q_{0} q_{-2}\right) l_{1}^{2}+\frac{1}{12}\left(3 l_{0} q_{2}+q_{3} q_{-1}+q_{1}^{2}+3 l_{1} q_{1}-q_{2} q_{0}\right) l_{-1}^{2} \\
& +\frac{1}{3}\left(-\frac{11}{20} l_{1} l_{-1}+l_{-1} q_{1}-\frac{3}{2} l_{0} q_{0}-l_{1} q_{-1}\right) q_{-3} q_{3}+\frac{1}{6}\left(\frac{1}{10} l_{1} l_{-1}-\frac{q_{-2} q_{2}}{6} l_{1} q_{-1}+\frac{2}{3} l_{0} q_{0}+\frac{1}{6} l_{-1} q_{1}\right) \\
& -\frac{1}{12}\left(l_{1} l_{-1}-\frac{34}{3} l_{0} q_{0}\right) q_{-1} q_{1}+\frac{1}{4}\left(l_{1} q_{2} q_{-3}-\frac{1}{9} l_{-1} q_{2} q_{-1}+\frac{1}{9} l_{1} q_{1} q_{-2}-l_{-1} q_{3} q_{-2}\right) q_{0}+\frac{q_{2}^{2} q_{-2}^{2}}{675} \\
& -\frac{1}{6}\left(9 l_{1} l_{-1}+l_{-1} q_{1}-l_{1} q_{-1}\right) l_{0} q_{0}+\frac{1}{12}\left(\left(q_{2} q_{-3}-q_{1} q_{-2}\right) l_{1} l_{0}+\left(-q_{3} q_{-2}+q_{2} q_{-1}\right) l_{-1} l_{0}\right)-l_{0} q_{0}^{3} \\
& +\frac{1}{18} l_{0} q_{2} q_{1} q_{-3}-\frac{1}{36}\left(q_{2}^{2} q_{-1} q_{-3}-l_{1} q_{3} q_{-2}^{2}+q_{3} q_{1} q_{-2}^{2}+l_{-1} q_{2}^{2} q_{-3}\right)-\frac{1}{9}\left(q_{1}^{3} q_{-3}+q_{3} q_{-1}^{3}\right)-\frac{5}{108} q_{1}^{2} q_{-1}^{2} \\
& +\frac{1}{5}\left(\frac{7}{6} q_{1} q_{-1}-3 q_{0}^{2}+\frac{1}{20} q_{2} q_{-2}\right) q_{-3} q_{3}+\frac{1}{18} l_{0} q_{3} q_{-1} q_{-2} .
\end{aligned}
$$

For this algebra, the transformations (19) defining the contraction $\mathfrak{g}$ are given by $L_{i}^{\prime}=L_{i}, Q_{\mu}^{\prime}=\frac{1}{t} Q_{\mu}$. The resulting algebra has an Abelian radical of dimension seven, which implies that the invariants will only depend on the $q_{\mu}$-variables [12]. It is straightforward to verify that $\mathcal{N}(\mathfrak{g})=4$, and from the four Casimir operators, two can be obtained by contracting the invariants $C_{2}$ and $C_{4}$ of $\mathfrak{s o}(5)$. A basis of invariants of $\mathfrak{g}$ is completed with two operators $C_{6}^{\prime}$ and $C_{8}^{\prime}$ of degrees 6 and 8 respectively. Omitting $C_{8}$ because of its length, the explicit form of the invariants $C_{2}^{\prime}, C_{4}^{\prime}$ and $C_{6}^{\prime}$ is as follows:

$$
\begin{aligned}
& C_{2}^{\prime}=-\frac{2}{5}\left(q_{3} q_{-3}+q_{1} q_{-1}\right)+\frac{1}{15} q_{2} q_{2}+q_{0}^{2} \\
& C_{4}^{\prime}=\frac{1}{6}\left(q_{3} q_{-1} q_{-2}+q_{2} q_{1} q_{-3}+\frac{1}{3} q_{1}^{2} q_{-2}+\frac{1}{3} q_{2} q_{-1}^{2}\right) q_{0}-\frac{1}{540}\left(q_{1} q_{-1}+36 q_{0}^{2}\right) q_{-2} q_{2} \\
& -\frac{1}{36}\left(q_{2}^{2} q_{-1} q_{-3}+q_{3} q_{1} q_{-2}^{2}\right)-\frac{1}{9}\left(q_{1}^{3} q_{-3}+q_{3} q_{-1}^{3}\right)+\frac{1}{5}\left(\frac{7}{6} q_{1} q_{-1}-3 q_{0}^{2}+\frac{1}{20} q_{2} q_{-2}\right) q_{-3} q_{3} \\
& +\frac{q_{2}^{2} q_{-2}^{2}}{675}-\frac{5}{108} q_{1}^{2} q_{-1}^{2}-\frac{3}{100} q_{3}^{2} q_{-3}^{2}, \\
& C_{6}^{\prime}=-729 q_{0}^{6}-54 q_{1}^{4} q_{-2}^{2}+54 q_{3} q_{-3}\left(9 q_{2} q_{0}^{2} q_{-2}+162 q_{1} q_{0}^{2} q_{-1}-32 q_{1}^{2} q_{-1}^{2}+6 q_{2} q_{1} q_{-1} q_{-2}\right) \\
& +6 q_{2} q_{-2}\left(6 q_{3} q_{-1}^{3}-10 q_{1}^{2} q_{-1}^{2}+6 q_{-3} q_{1}^{3}-63 q_{1} q_{0}^{2} q_{-1}\right)-162 q_{0}^{2}\left(q_{-2}^{2} q_{3} q_{1}+q_{2}^{2} q_{-3} q_{-1}\right) \\
& +54\left(q_{0}^{2}\left(27 q_{3}^{2} q_{-3}^{2}-8 q_{-3} q_{1}^{3}-8 q_{3} q_{-1}^{3}-13 q_{1}^{2} q_{-1}^{2}\right)-q_{3}^{2}\left(-q_{0} q_{-2}^{3}+q_{-1}^{2} q_{-2}^{2}\right)-\left(q_{1}^{2} q_{-3}^{2}+q_{-1}^{4}\right) q_{2}^{2}\right) \\
& +972\left(q_{0}^{3}\left(q_{3} q_{-1} q_{-2}+q_{2} q_{1} q_{-3}\right)-\left(q_{3}^{2} q_{-1} q_{-2} q_{-3}+\left(q_{2} q_{-1}^{2} q_{-3}+q_{2} q_{1} q_{-3}^{2}+q_{1}^{2} q_{-2} q_{-3}\right) q_{3}\right) q_{0}\right) \\
& +288 q_{-1} q_{1}\left(q_{-3} q_{1}^{3}+q_{3} q_{-1}^{3}\right)+90 q_{-2} q_{2}\left(q_{1}^{2} q_{-2}+q_{2} q_{-1}^{2}\right) q_{0}+396 q_{-1} q_{0} q_{1}\left(q_{1}^{2} q_{-2}+q_{2} q_{-1}^{2}\right) \\
& +180 q_{1} q_{-1}\left(q_{-2}^{2} q_{3} q_{1}+q_{2}^{2} q_{-3} q_{-1}\right)+864 q_{-3} q_{3}\left(q_{-3} q_{1}^{3}+q_{3} q_{-1}^{3}\right)+q_{2}^{3} q_{-2}^{3}-64 q_{1}^{3} q_{-1}^{3}+q_{2}^{3} q_{0} q_{-3}^{2} \\
& -324 q_{0}^{3}\left(q_{1}^{2} q_{-2}+q_{2} q_{-1}^{2}\right)-18 q_{-2} q_{2}\left(q_{-2}^{2} q_{3} q_{1}+q_{2}^{2} q_{-3} q_{-1}\right)-756 q_{0} q_{1} q_{-1}\left(q_{3} q_{-1} q_{-2}+q_{2} q_{1} q_{-3}\right) \\
& +243\left(6 q_{1} q_{-1}-30 q_{3} q_{-3}+q_{2} q_{-2}\right) q_{0}^{4}-3 q_{2}^{2} q_{-2}^{2}\left(4 q_{1} q_{-1}+9 q_{0}^{2}\right)
\end{aligned}
$$

By inspection, we easily see that $C_{2}-C_{2}^{\prime}=C_{21}$, therefore the set $\left\{C_{2}, C_{4}, C_{21}, C_{2}^{\prime}, C_{4}^{\prime}, C_{6}^{\prime}\right\}$ has at most rank five. Computing the Jacobian with respect to the variables $\left\{q_{-3}, q_{0}, q_{1}, l_{1}, l_{0}\right\}$, we prove that the rank is indeed five. We can therefore solve the missing label problem. From the preceding functions we deduce that a missing label operator is at least of order 4 , thus reconfirming the observation on the minimal 
Table 2. $\mathfrak{s o}(5)$ brackets in a $\mathfrak{s o}(3)=\left\{L_{0}, L_{ \pm 1}\right\}$ basis.

\begin{tabular}{c|ccccccc}
{[]} & $Q_{3}$ & $Q_{2}$ & $Q_{1}$ & $Q_{0}$ & $Q_{-1}$ & $Q_{-2}$ & $Q_{-3}$ \\
\hline \hline$L_{0}$ & $3 Q_{3}$ & $2 Q_{2}$ & $Q_{1}$ & 0 & $-Q_{-1}$ & $-2 Q_{-2}$ & $-3 Q_{-3}$ \\
$L_{1}$ & 0 & $6 Q_{3}$ & $Q_{2}$ & $2 Q_{1}$ & $6 Q_{0}$ & $10 Q_{-1}$ & $Q_{-2}$ \\
$L_{-1}$ & $Q_{2}$ & $10 Q_{1}$ & $6 Q_{0}$ & $2 Q_{-1}$ & $Q_{-2}$ & $6 Q_{-3}$ & 0 \\
$Q_{3}$ & 0 & 0 & 0 & $Q_{3}$ & $Q_{2}$ & $10 Q_{1}+15 L_{1}$ & $5 Q_{0}-15 L_{0}$ \\
$Q_{2}$ & & 0 & $-6 Q_{3}$ & $-Q_{2}$ & $-15 L_{1}$ & $30 Q_{0}+60 L_{0}$ & $10 Q_{-1}-15 L_{-1}$ \\
$Q_{1}$ & & & 0 & $3 L_{1}-Q_{1}$ & $-3 L_{0}-3 Q_{0}$ & $15 L_{-1}$ & $Q_{-2}$ \\
$Q_{0}$ & & & & 0 & $-Q_{-1}-3 L_{-1}$ & $-Q_{-2}$ & $Q_{-3}$ \\
$Q_{-1}$ & & & & & 0 & $-6 Q_{-3}$ & 0 \\
$Q_{-2}$ & & & & & 0 & 0 \\
\hline \hline
\end{tabular}

degree of such an operator made in 29]. This fourth order operator can be taken for example as $\Phi_{1}=C_{4}-C_{4}^{\prime}+\frac{7}{12} C_{21}\left(C_{21}-C_{2}\right)$. We point out that this choice does not coincide with that made in [29], where the simplest possible fourth order operator was considered. A sixth degree missing label operator that commutes with $\Phi_{1}$ can be taken as $\Phi_{2}=C_{6}^{\prime}-13608 C_{4}\left(C_{2}-C_{21}\right)+729\left(C_{2}^{2}-C_{21}^{3}\right)+2187\left(C_{2}^{2}-C_{21}^{2}\right)$.

\section{On the validity of the method}

The contraction method can constitute a practical procedure to reduce to some extent the computations when we consider reduction chains $\mathfrak{s} \supset \mathfrak{s}^{\prime}$ with more than three missing labels, whenever the conditions of theorem 1 are satisfied. For example, a solution for the general chains $\mathfrak{s p}(2 N) \supset \mathfrak{s p}(2 N-2) \times \mathfrak{u}(1)$ or $\mathfrak{s p}(2 N) \supset \mathfrak{s p}(2 N-2) \times \mathfrak{s u}(2)$, considered for the first time in [30], can be found by analyzing the corresponding contractions.

As has been pointed out when deriving formula (30), the contraction method could fail if the contraction $\mathfrak{g}$ has "to few" invariants with respect to the number of necessary labelling operators. Actually, this can happen for reductive $\mathfrak{s}^{\prime}$ and semisimple $\mathfrak{s}$ if the following numerical equality $\mathcal{N}(\mathfrak{s})=\mathcal{N}(\mathfrak{g})=n$ holds. Since in this case a fundamental system of invariants of the contraction $\mathfrak{g}$ can be obtained by appropriate contraction of the Casimir operators of $\mathfrak{s}$, the dependence of the quadratic Casimir operator implies that we get at most $n-1$ of the needed labelling operators. The remaining operator, which must be computed explicitly, may however be determined in some sense by the other operators, by means of the commutation property it must satisfy. Although for this extreme case we don't obtain a complete set by the contraction, it could also happen that any degeneracy of practical interest can be resolved using only the $n-1$ operators associated to the contraction. This however requires a case by case inspection.

The lowest dimensional reduction where the contraction produces an insufficient number of labelling operators is the reduction $G_{2} \supset \mathfrak{s u}(2) \times \mathfrak{s u}(2)$, where $G_{2}$ is the exceptional Lie algebra of rank two. In this case, we have $n=2$ missing labels, therefore four available operators. In [31], a pair of commuting operators of order six that solves the missing label problem was found. The general form of commuting operators remains 
however an unanswered question. Observe that here, $\mathcal{N}\left(G_{2}\right)=\mathcal{N}\left([\mathfrak{s u}(2)]^{2}\right)=2$ holds. In this case, the $G_{2}$ generators decompose as those of the subalgebra and an eight dimensional irreducible representation $R$ of $\mathfrak{s u}(2) \times \mathfrak{s u}(2)$, therefore the contraction has the Levi decomposition $\mathfrak{g}=(\mathfrak{s u}(2) \times \mathfrak{s u}(2)) \vec{\oplus}_{R} 8 L_{1}$. This algebra satisfies $\mathcal{N}(\mathfrak{g})=2$. This means that the invariants $C_{2}^{\prime}$ and $C_{6}^{\prime}$ of the contraction algebra are obtained by limiting procedure from the quadratic and hexic Casimir operators of $G_{2}$. Now the quadratic operator is dependent on the operators of the same degree of $G_{2}$ and the subalgebra. A routinary but cumbersome computation shows that the function $C_{6}^{\prime}$ is independent on the invariants of the algebra-subalgebra chain. Therefore we arrive at a missing label operator $\Phi$ of degree six, but a second independent operator cannot be constructed, because there is no other independent higher order invariant in the contraction. Taking into account the construction made in [31], this second operator must be either of degree six or eight. Since both $G_{2}$ and $\mathfrak{g}$ have at most one (independent) invariant of order higher than two, the failure of the contraction seems to be directly related to the order of the required labelling operators.

\section{Conclusions}

We have shown that many physically relevant missing label problems can be completely solved by using the properties of the reduction chain $\mathfrak{s} \supset \mathfrak{s}^{\prime}$, by means of a Lie algebra contraction associated to this reduction. Analyzing the set of invariants of the three involved Lie algebras, suitable commuting operators can be found that solve the missing label problem. In this approach, the found operators inherit an intrinsic meaning, namely as those terms of the Casimir operators of $\mathfrak{s}$ that get lost during contraction, up to some combination of lower order invariants of $\mathfrak{s}$ and $\mathfrak{s}^{\prime}$. We have recomputed some classical reductions appearing in atomic and nuclear physics, obtaining complete agreement with the result obtained by different authors and techniques. Further we have furnished a natural explanation of the order of these operators, which are directly related to the order of the Casimir operators of the contracted Lie algebra. For the special case of $n=m=0$, we have obtained a direct relation among the invariants

of $\mathfrak{s}$ and $\mathfrak{s}^{\prime}$ with those of the contraction $\mathfrak{g}$, which provides a new interpretation of the contracted invariants.

It seems natural that, whenever the reduction chain is non-canonical and the reduction is not multiplicity free, the information lost is somehow determined by the chain itself, and not by a priori external techniques. In this sense, the missing label operators which arise from the contraction $\mathfrak{g}$ should correspond to the natural choice of physical labelling operators, as they are obtained using only the available information on the algebra-subalgebra chain and their invariants. This suggests that these could be the correct physical operators to be considered for the labelling of states. An argument supporting this interpretation is the equivalence of the contraction procedure with the Kmatrix method in the $\mathfrak{s u}(3) \supset \mathfrak{s o}(3)$ chain or the Wigner supermultiplet model. Whether the remaining possibilities that arise from the general algebraic solution of the missing 
label problem are physically more relevant than those operators found by contraction, remains a question that should be analyzed for any specific physical situation. All examples show also that the affine contraction provides at most $n$ of the $2 n$ available operators, thus induces a kind of partition in the set of labelling operators. This suggests the existence of a certain kind of hierarchy among these operators, as well as the fact that some of them are not directly related to the properties of the embedding of the subalgebra, and therefore not equivalent to these. The next natural step is to analyze if the contraction $\mathfrak{g}$ can also be used to derive the eigenvalues of the missing label operators.

The failure of the proposed method for the special case $\mathcal{N}(\mathfrak{g})=\mathcal{N}(\mathfrak{s})=n$ is essentially a consequence of the existence of the quadratic Casimir operators for reductive Lie algebras. In this situation, a similar obstruction to obtain the sufficient number of labelling operators will appear whenever the Lie algebra $\mathfrak{s}$, the subalgebra $\mathfrak{s}^{\prime}$ and the contraction $\mathfrak{g}$ have all a Casimir operator of the same degree. In this case the invariant of the contraction will be dependent, we thus loose one solution. How to recover this operator without solving explicitly the system of partial differential equations remains unanswered, as well as the meaning of this lost solution. In spite of this incompleteness, the method is still worthy to be applied, since often particular degeneracies can be solved using less than the required labelling operators [32].

Finally, the contraction method, essentially reducing the obtainment of missing label operators to the computation of invariants of three Lie algebras, constitutes an appropriate class of algebras to be tested with the geometrical method based on moving frames, recently introduced in [33, 34], and tested successfully for large types of algebras. In this frame, the solving of differential equations is replaced by algebraic systems,

which can be often be solved in more effective manner. This algorithm can be therefore applied more efficiently to obtain a maximal number of independent invariants of the three Lie algebras involved in the MLP. Further, this approach probably allows to deduce some properties linking the corresponding automorphism groups of these Lie algebras. Moreover, in the case of non-reductive subalgebras, the geometric method provides solutions avoiding complex realizations of the invariants, therefore discarding supplementary complications that usually arise from the analytical approach. Whether the method can be implemented to compute directly the missing label operators, is a problem that has still to be explored.

\section{Acknowledgement}

The author would like to thank the Universidad Nacional de Rosario, where part of this work was done, as well as H. de Guise for helpful discussions and additional references. This work was partially supported by the research grant MTM2006-09152 of the Ministerio de Educación y Ciencia. 


\section{References}

[1] Elliott J P 1958 Proc. Roy. Soc. Lond. A 245 128, 562

Racah G 1949 Phys. Rev. 761352

[2] Rowe D J 1995 J. Math. Phys. 361520

[3] Rowe D J, Le Blanc R and Repka J 1989 J. Phys. A: Math. Gen. 22 L309

[4] Sharp R T 1975 J. Math. Phys. 16, 2050

[5] Peccia A and Sharp R T 1976 J. Math. Phys. 171313

[6] Celeghini E, Tarlini M and Vitiello G 1984 Nuovo Cimento A 8419

[7] Racah G 1951 Group Theory and Spectroscopy (Princeton Univ. Press, N. J.)

[8] Patera J, Sharp R T, Winternitz P and Zassenhaus H 1976 J. Math. Phys. 17986

[9] Campoamor-Stursberg R 2006 SIGMA 2 p28

[10] Segal I E 1951 Duke Math. J. 18221 Inönü E and Wigner E P 1953 Proc. Nat. Acad. Sci U.S.A. 39510

[11] Weimar-Woods E 2000 Rev. Math. Phys. 121505

[12] Campoamor-Stursberg R 2003 J. Phys. A: Math. Gen. 361357

2003 Acta Physica Polonica B 343901

Ndogmo J C 2004 J. Phys. A: Math. Gen. 375635

[13] Weimar-Woods E 1996 Proc. XXI Int. Colloq. Group Theoretical Methods in Physics (Goslar), vol 1, (Singapore: World Scientific) p 132

[14] Campoamor-Stursberg R 2007 Acta Phys. Pol. B 383

[15] Dynkin E P 1952 Mat. Sb. 30349

Gruber B and Lorente M 1972 J. Math. Phys. 131639

[16] Patera J and Sankoff D 1973 Tables of Branching Rules for Representations of Simple Lie algebras (Montréal: Presses de l'Université de Montréal)

[17] Chaichian M, Demichev A P and Nelipa N F 1983 Comm- Math. Phys. 90353

[18] Herranz F J and Santander M 1997 J. Phys. A: Math. Gen. 305411

Herranz F J, Perez Bueno J C and Santander M 1998 J. Phys. A: Math. Gen. 315327

[19] Campoamor-Stursberg R 2006 J. Phys. A: Math. Gen. 392325

[20] Campoamor-Stursberg R 2007 J. Phys. A: Math. Theor. 405355

[21] Louck J D 1976 Proceedings of the International Symposium on Mathematical Physics, Mexico City, Mexico

[22] Judd B R, Miller W, Patera J and Winternitz P 1974 J. Math. Phys. 151787

[23] Bargmann V and Moshinsky M 1965 Nucl. Phys. 23177

[24] Hecht K T 1965 Nucl. Phys. 63177

[25] Sharp R T and Pieper S C 1968 J. Math. Phys. 9663

[26] Quesne Ch 1976 J. Math. Phys. 17, 1452; 18, 1210 (1977).

[27] Partensky A and Maguin C 1978 J. Math. Phys. 19511

[28] Moshinsky M and Nagel J G 1963 Phys. Letters 5173

[29] De Meyer H, Vanden Berghe G, Van der Jeugt J and De Wilde P 1985 J. Math. Phys. 262124

[30] Bincer A M 1980 J. Math. Phys. 21671

-1983 J. Math. Phys. 241695

[31] Hughes J W B and Van der Jeugt J 1985 J. Math. Phys. 26894

[32] Giroux Y and Sharp R T 1987 J. Math. Phys. 281671

[33] Boyko V, Patera J and Popovych R 2006 J. Phys. A: Math. Gen. 395749

[34] 2007 J. Phys. A: Math. Theor. 40113 